\title{
UTEROTUBAL INSUFFLATION AND HYSTEROSALPINOGRAPHY
}

\author{
By PERCY MALPAS, Ch.M., F.R.C.S., F.R.C.O.G. \\ (Honorary Surgeon, The Women's and Maternity Hospitals, Liverpool)
}

Although during the past twenty-five years insufflation and hysterosalpingography have both become established and widely practised procedures in the investigation of sterility, there are still certain aspects of both techniques upon which full agreement has not been reached. One of these is the question whether they should be regarded as alternative rather than as complementary procedures, another the determination of their true therapeutic as opposed to their undoubted diagnostic value. There is, too, an even more pressing need to integrate both procedures with the newer methods now available for the investigation of sterility.

In the preparation of the present paper much help has been derived from the recent paper by Sharman (I944), particularly with regard to the technique and results of insufflation, and the papers by Witwer, Bushman and Leucutia (I930), and Green-Armytage (I943) dealing with hysterosalpingography. In order to provide a personal standard of reference a special re-examination and follow-up was undertaken of cases investigated in the five years preceding the war. This period was chosen in order to allow a reasonable time for the end results to be apparent, and also to avoid the complications introduced by the war. In so complex a problem as sterility it is desirable to deal with the most stable population possible.

\section{Definition of Terms.}

At the outset some consideration of the nomenclature of the problem seems advisable. It is a sound general principle that when any complex biological end-problem is being examined no terms should be used which may prejudge the causes. Neglect of this may lead to serious misjudgment of the value of any particular mode of treatment. There is accordingly much to be said in favour of calling the particular problem which is now under consideration "childlessness" rather than "sterility," and apart from its greater scientific accuracy such a change in terminology carries certain other advantages. For one thing, it does not place the stigma of sterility on couples who may be perfectly normal and are childless for some random and perhaps temporary cause. The point is not unimportant, since conception, depending as it does on intercourse, is in turn conditioned by psychological factors. Again, such a change in nomenclature would obviate the not uncommon cases when a couple dubbed sterile unexpectedly have a child, often after they have adopted one.

To put the problem in another way, the initial step in the investigation of childlessness is to pick out the cases of sterility, i.e. those cases in which conception is impossible, either by reason of a gross seminal defect, permanent absence of ovulation, or permanent occlusion of the female genital tract, leaving a group of cases in which our knowledge of the cause of the childlessness is deficient, a group in which the causes may be transient and in which the prognosis is good.

\section{Order of Examination.}

Ideally both the husband and the wife should be investigated, but circumstances may prevent this. With most couples it is easier to investigate the husband first; sometimes however, when the man will not co-operate, it is quite reasonable to confine the examination to the wife. The evidence that any given woman is producing ova is still purely inferential in contrast with the direct evidence given by sperm examination, and there are certainly some childless marriages in which it may be better to leave the responsibility for the childlessness unallocated after taking steps to assure the woman that there is no evident bar to conception.

\section{Indication for Investigation of Tubal Patency}

Until means were available for determining tubal patency all studies of human infertility were vitiated by the fact that no clinical method can detect every case of tubal occlusion. The Fallopian tubes may be blocked by an inflammatory process so mild or transient that no evidence whatever is left behind, on the other hand, though not so frequently, a gross and unmistakable appendage inflammation does not certainly preclude conception. It was not until the two 
techniques of insufflation, introduced by Rubin in 1920 and lipiodal hysterosalpingography, a measure of more complicated a parentage, but probably first introduced with precision by Heuser in I92I, were developed that a start could be made in the unravelling of the tangle of the factors determining childlessness.

No arbitrary rules can be laid down as to when a childless marriage should be investigated. Rubin, in considering the criteria by which the success of insufflation may be measured, gave an age level of thirty years, a duration of marriage of three years, and a period of at least one year without contraception prior to the insufflation. To these criteria certain objections may be raised. In some ways the age level is too high. Many couples are disturbed about their childlessness before the woman has reached the age of thirty, and in view of the fact that successful treatment becomes less likely as the woman grows older there is much to be said for not adopting any arbitrary age level as an indication for investigation. On the other hand, many would consider a year only without contraception too short a time. The chances of conception from any single coitus are so low that a year may easily pass without pregnancy occurring in perfectly normal marriages. When this point is under consideration in any given case it is most essential to determine the total duration of cohabitation, which under modern conditions often bears a low ratio to the total duration of the marriage. In general, it would be agreed that any childless marriage in which the cohabitation period totals two to three years should be investigated.

\section{Contraindications to Investigation}

The contraindications to uterotubal insufflation and hysterosalpingography are similar and may be considered together. In the presence of inflammatory appendage disease or its sequelae neither procedure should be carried out except after very serious consideration, and then only if a vigorous bimanual examination and a course of short-wave pelvic diathermy have demonstrated the complete quiescence of the inflammation. The risk is no academic consideration. In ten years I have had, despite every reasonable care, two personal cases of pelvic peritonitis following insufflation, one needing abdominal drainage, and have seen three similan cases under the care of colleagues, one of them fatal. A sound if somewhat paradoxicat clinical rule is to exercise extra caution in deciding upon an insufflation in any case where there is reason to suspect the test will be negative. The occasional occurrence of these complications is a real objection to doing routine insufflations on ambulatory patients.

The risk of inflammatory reactions are admittedly higher after hysterosalpingography than after insufflation, but this may very likely be due to the fact that hysterosalpingography is usually performed in cases where a prior insufflation has revealed tubal occlusion.

\section{UTEROTUBAL INSUFFLATION}

Several types of apparatus are available. The differences essentially concern the calibre of the insufflation cannula, the method adopted for registering the pressure, whether by a manometer or a kymograph, and the presence or absence of any device for limiting the volume of gas injected.

As regards the calibre of the cannula the narrow bore cannula devised by Rubin requires no initial dilatation of the cervix, and its use does not necessarily call for anaesthesia. These advantages are offset by the fact that a higher proportion of erroneous negative results is obtained than when the wider cannula of Bonney's apparatus is employed. Even with a wide bore cannula a negative result can be often changed to a positive by a little further dilatation. From a personal experience of both cannulae I have found the wide bore of Bonney's cannula the more satisfactory.

The advantages of a kymographic method of recording the pressure are that the minor waves of the tubal contractions can be observed, it is rather easier to pick out the cases of tubal spasm and tubal stenosis, and a record of the result is available for preservation. On the other hand the apparatus is certainly more complex, and it is still a debatable point whether the minor waves due to the tubal contractions have any diagnostic or inferential value. Sharman dealing with this point states that these waves cannot be correlated with the phases of endometrial or ovarian activity. Certainly for any formal investigation of the causes of sterility an instrument such as Bonney's has every advantage; for ordinary clinical work the simple manometric apparatus of Bonney is quite adequate. 
With regard to the question whether the instrument should incorporate any method for limiting the volume of gas injected, as for instance is provided in Provis's apparatus, it would seem that the risks of air embolism depend more on the injection pressure than upon the volume injected.

\section{Time of Performance of the Tests.}

Formerly insufflation was usually performed in the first half of the menstrual cycle, preferably between the 4th and the Ioth day after the end of the periods in order to minimise the chance of obtaining an erroneous result through endometrial hyperplasia occluding the tube, and also to avoid the rather academic risk of transplanting endometrium upwards into the tubes. With the increasing importance of determining the presence of ovulation by the appearance of secretory changes in the endometrium there is much to be said for choosing the premenstrual phase so that a biopsy may be done at the same time.

The best time for hysterosalpingography also seems to be later in the cycle than was formerly considered most suitable. Rohan Williams (I944) in a paper describing five cases of venous intravasation emphasises that hysterosalpingography should not be done until menstrual regeneration is complete after menstruation, i.e. not before the eighth post-menstrual day and after the trauma of any previous curettage or dilatation is healed.

\section{Anaesthesia.}

If an initial dilatation is to be done, then anaesthesia is necessary. With a narrow bore cannula and a willing patient it is not essential, though sometimes the manœuvres necessary to close the cervix efficiently round the cannula may be painful. According to most authorities anaesthesia does not appear to modify either the response to the test or the escape pressure of the gas.

\section{Insufflation-Operative details.}

I. A bimanual examination is made of the appendages to check any previous findings.

2. The apparatus is tested for leaks.

3. The cervix is grasped with vulsella. These must be efficient and selected with care, as many vulsella on the market are unsatisfactory. The teeth must be deep enough to bite on to the fibrous stroma beneath the cervical skin, but not so massive as to injure the cervix; there should be no side play between the teeth when traction is made and the curve must be suitable to keep the shanks out of the way of the insufflation cannula. In the common cases of cervical anteflexion it is sometimes difficult to grasp the anterior lip efficiently, and Brigg's side-grip vulsella are often useful.

4. After dilatation to about I4 (Hawkins-Ambler) the cannula is inserted to its maximum extent and the cervix closed round it by applying one or two more vulsella.

5. The uterus is displaced upwards, and the vagina filled with water to detect leakage.

6 . The pressure is then steadily raised, the manometer watched for the escape threshold, and the type of response noted. The pressure should not be allowed to rise above 2 Io mms. $\mathrm{Hg}$, the approximate threshold of tissue and vascular air intravasation.

7. If a negative result is obtained the cervix should be dilated further up to No. 20 dilator (Hawkins-Ambler) and the test repeated. It is, of course, important to remain content with a negative result on the second attempt, and dangerous to attempt to get a positive result by raising the pressure above the safety level.

\section{Insufflation Findings.}

I. Normal patency - the pressure reaches 60 to $\mathrm{I} 20 \mathrm{mms}$. $\mathrm{Hg}$ and then falls rapidly.

2. Non-patency.

3. Spasm-the escape pressure is high, the flow does not take place at once, but when it does so its fall to zero pressure is rapid. A repetition of the test a few minutes later shows normal patency, a sign that the spasm has disappeared.

4. Stenosis - the escape pressure is high, the fall is slow, and repetition of the test gives a similar result. 


\section{Significance of a Negative Result.}

In a small proportion of cases insufflation certainly gives an erroneous result. Sharman found 6 cases patent to hysterosalpingography among 60 cases non-patent to insufflation, and $\mathbb{D}$ among his II6 cases who became pregnant there were 29 who had been diagnosed as having $c$ closed tubes as a result of a single instufflation. In a smaller personal series of I2 negative in- $\vec{\Rightarrow}$ sufflation followed up for four years, pregnancy occurred in one instance.

\section{Pregnancy following Insufflation}

In the five years up to the outbreak of war 147 childless marriages of three years duration or more were personally investigated. Owing to war drift of population only 66 of these could be traced in I944. In twelve cases insufflation was negative, pregnancy subsequently occurring in one of these.

Of the 44 positive cases two wives lost their husbands soon after the test, one separated from her husband, and in a fourth case later examination of the husband showed azoospermia.

Among the remaining 40 positive cases 26 pregnancies occurred, approximately 65 per cent.

At first sight this figure seemed strong evidence of the therapeutic value of a positive insufflation. A closer examination, however, of the cases revealed the rather disconcerting fact that most of the conceptions occurred not soon after the test, but one or two years later. Only 6 of the 26 children were born within the year, 13 were born within two years, and 7 were born in the third year or later. This finding, which would seem to cast doubt upon the immediate therapeutic value of insufflation, is at variance with some of the published results. Rubin $N$ (I939), for instance, found that 68 per cent of his successes became pregnant within six months. Sharman gives a lower figure, 36 per cent of his III pregnancies commencing within six months. It may be that the present series is very small, too small to draw any precise conclusions, but I believe such a finding of the delay in conception accords with general clinical experience. To a certain extent the problem cannot be solved by statistics, it is far too complex. For one thing $\frac{2}{\infty}$ any couple so desirous of children as to have their childlessness investigated are making every effort. Perhaps the following point has a bearing on this problem of the therapeutic value of $\oplus$ the test.

Insufflation is a test carried out on the female partner. If it has any therapeutic valus one would expect its success to be qualified by the age of the woman, because it is she who is being tested, and not by the duration of the marriage, which introduces the factor which is not under test, namely, the husband. The results of the present series, admittedly a small one, give an opposite finding. The average age of the successful group was $29 \cdot 4$, that of the unsuc- $\mathbb{D}$ cessful 3I, very little more. On the other hand there was a big difference between the average duration of marriage in the successful group and that of the unsuccessful group, 4.08 years as $\frac{0}{3}$ compared with 6.5 years. In other words, the apparent therapeutic value of insufflation may depend on the normal chances of spontaneous conception, chances which clearly fall more rapidly with each successive year of marriage than with each increasing year of the woman's age.

\section{HYSTEROSALPINGOGRAPHY}

Generally hysterosalpingography has been regarded as a complement to insufflation, for use in cases where insufflation is negative and with the twofold object of confirming the occlusion and if possible to determine its site. For this reason it is easy to see why the proportion of 0 inflammatory reactions is higher after hysterosalpingography than after insufflation. The question may be asked to what extent it should be used as an alternative procedure. It certainly $\frac{D}{2}$ does away with the risks of air embolism. C. H. Walsh has described a fatal case of a woman insufflated on the day before the period was due, and he was able to find records of six similar $\mathscr{N}$ cases in the literature. On the other hand, air embolism is a very rare accident, and the risks N of its occurrence scarcely offset the advantages of the simplicity and economy of air insufflation. N As regards the fallacies of either methods there is no doubt that both sometimes give erroneous $\omega$ negative results, though perhaps the advantage in this respect lies with the radiological method.

\section{Technique of Uterosalpingography.}

The test should not be performed in the first week after the period has finished, nor in the few days before the next period is due. The morning of the test the lower bowel should be 
PLATE I

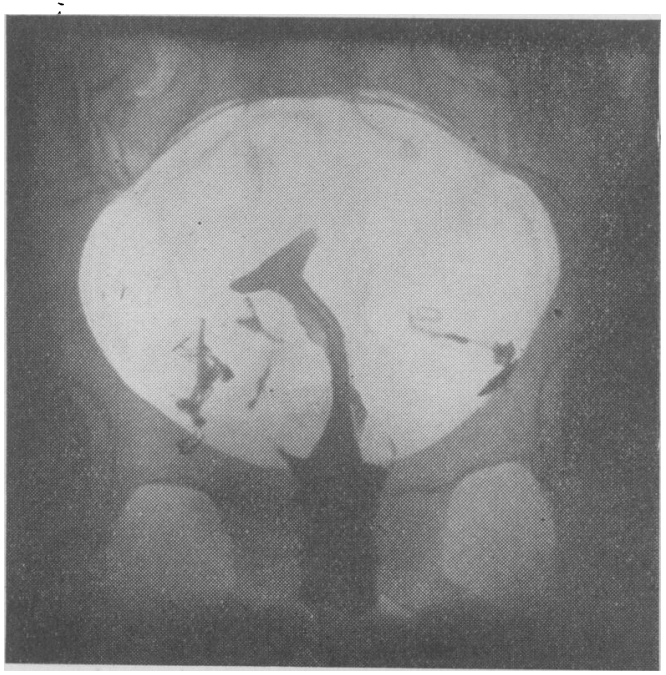

Normal Patency

PLATE III

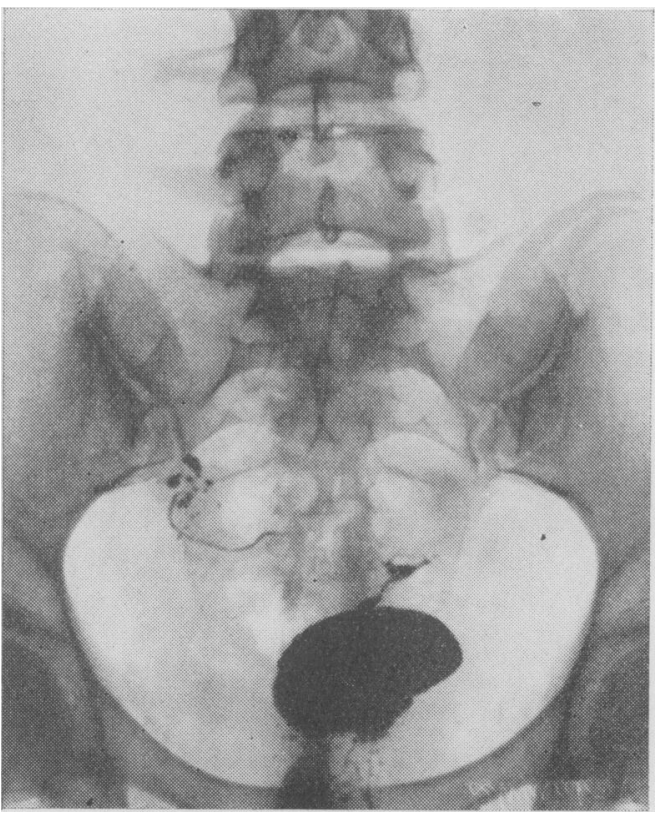

After Right Salpingostomy Tubal Patency
PLATE II

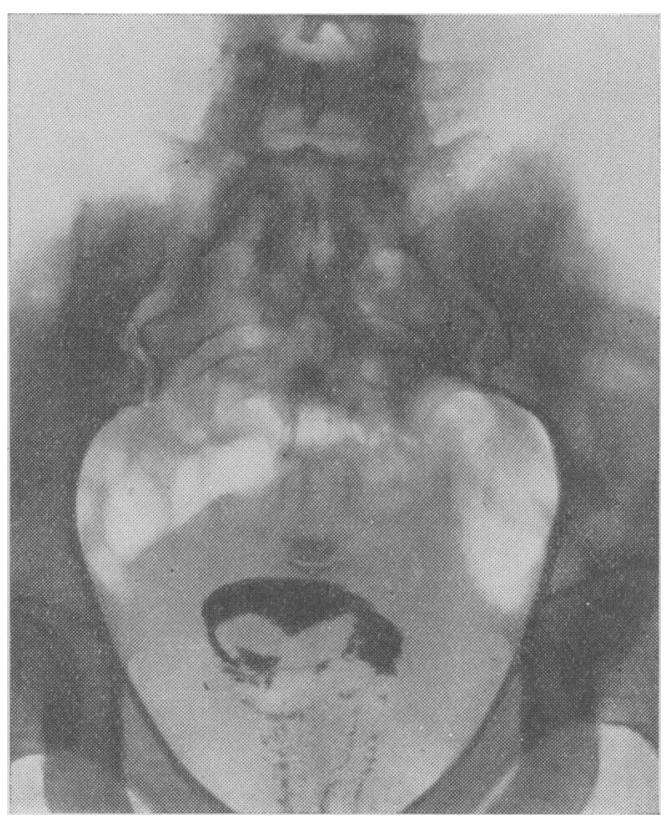

Tubal Occlusion

PLATE IV

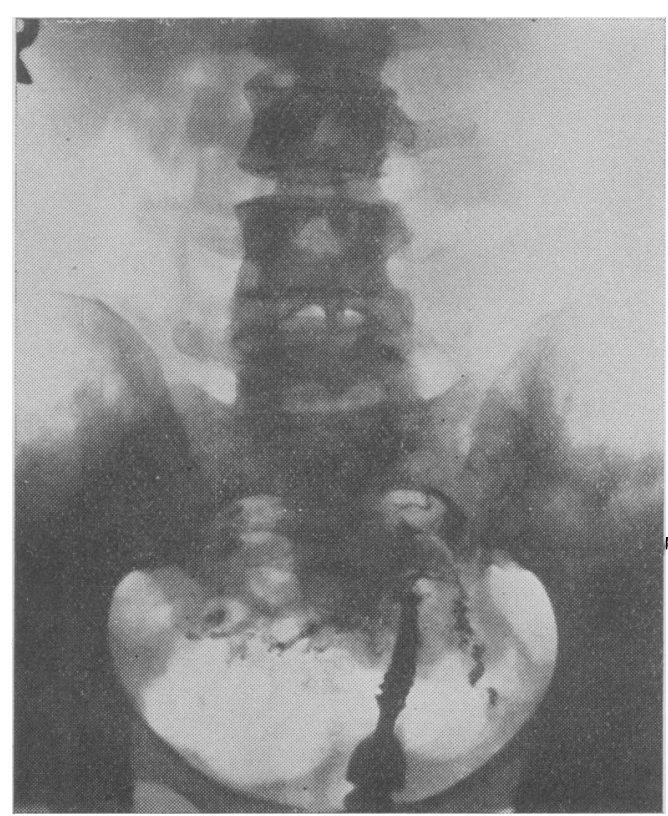

VENOUS INTRAVASATION 
emptied by an enema to reduce adventitious shadows. Anaesthesia is usually unnecessary, although, if the patient is likely to resent instrumentation, it is better to use an anaesthetic than to obtain a confused result from a technique muddled by the patient's resistance.

The injection itself is best done over a screen. Otherwise the interpretation is difficult and may be erroneous. If the lipiodal is seen entering and then spilling from the fimbriated ends a precise and positive observation has been made which is far superior to that given by inspection of films with their many shadows.

After exposing the cervix a cannula with an olive obturator is inserted as far as the internal os. Green Armytage has emphasised the need for the use of a cannula with a distal aperture so as to allow the easy passage of the fluid into the uterus. The cannula is held firmly by countertraction on the cervix with vulsella and the oil, at a temperature of $105^{\circ} \mathrm{F}$., is slowly injected with a record syringe, only sufficient pressure being exerted to move the column of oil just perceptibly. After 2 c.c.s have been injected the patient may complain of slight cramping pains. If the piston tends to rebound at this stage, it is a sign that the uterus is filled, and a wait is advisable. A further injection is then attempted, but if the rebound persists the injection must be regarded as complete. The amount of oil that is usually needed varies between $2 \cdot 5 \mathrm{c}$.c. and 5 c.c., and it is better to use too little than too much.

As soon as the oil is seen escaping from the fimbriated ends any excess in the vagina is wiped away, a little gauze packing placed round the cervix to hold the cannula in place, and an X-ray film is taken. In cases where there is any doubt about the tubal patency it is advisable to take a second film 6 to $\mathrm{I} 2$ hours later. If the tube is patent, its musculature will during this period extrude the oil into the peritoneal cavity.

Radiological Appearances (see Plates I, 2, 3, 4).

The uterus is readily seen as a wedge-shaped shadow. The tubes if normal will appear as fine lines increasing in thickness toward the fimbriated ends (Plate I) Peritoneal spill, the only sure sign of patency, is indicated by the presence of smooth globular shadows in the pelvis. Plate 2 shows a negative result, the tubes being occluded at the uterine end, the commonest site. Plate 3 shows the appearances after a bilateral salpingostomy. On one side the tube is occluded, on the other it is patent, but the straightness of the shadow of the filled tube demonstrates the rigidity of its wall as compared with that of a normal tube. The present paper is perhaps not the place to discuss the value of salpingostomy, but it must be emphasised that patency alone is not the sole criterion of a healthy tube. Parker has shown that the passage of the spermatozoa along the tube depends largely on capillary currents passing along the intercommunicating longitudinal channels formed by the folds of the mucous membrane, these currents being set in motion by the ciliae of the lining cells. If the plicae are flattened or adherent a tube may be non-functional, though perfectly patent. This is the likely reason why most surgeons are disappointed with the results of their salpingostomies, and it is rather difficult to reconcile the reports of enthusiasts for this operation with the general experience.

\section{Fallacies of Hysterosalpingography}

Hysterosalpinography is perhaps a more reliable test than insufflation, but all the same it is not infallible. Interpretation of the films is difficult, and for this reason screen examination is desirable. Sharman, discussing the point, states that hysterosalpingography may show blockage where none exists, and its interpretation may be so difficult that an accurate conclusion cannot be reached.

Venous intravasation of the injected fluid is not uncommon. Fortunately the accident does not appear to be followed by ill-effects, and its main disadvantage is that it confuses the radiological appearances. Plate 4 , from a film kindly lent me by Dr. C. H. Walsh, illustrates the appearances. The intravasation picks out the smaller veins radiating from the uterus as a cluster of fine lines, or sometimes shows up the whole or part of the uterine or the ovarian veins. The risks of intravasation may be minimised by doing the test only after the eighth postmenstrual day, and taking care not to inject too much at too high a pressure.

\section{Results}

The proportion of pregnancies following hysterosalpingography depends on whether it is used as a routine procedure, in which case the results are very similar to those following routine 
insufflation, or whether it is used as a complementary measure in cases of negative insufflation, in which the proportion of pregnancies will be much lower. There has in fact been no satisfactory explanation given for the supposed therapeutic value of either insufflation or hysterosalpingography. It may indeed be that the reason for this is that no such value exists, a point discussed earlier. Spasm and plugs of secretion may be removed; these are on the other hand essentially transient lesions which would probably pass away in any case. Perhaps the dilatation of the tube may reflexly stimulate the ovary to ovulate, but of such an effect there is no evidence. If scar tissue following an infection is really present it is difficult to imagine it being relieved by lipiodal in view of the unfavourable results experienced in other organs in the removal of adhesions, and Sharman was unable to find that repeated insufflation ever opens up a tube which has been shown to be occluded.

\section{SUMMARY AND CONCLUSIONS}

Fundamentally cases of childless marriage can be resolved into two groups, one comprising those cases in which conception is impossible, the sterile group, the other comprising those cases in which no cause for the failure to conceive can be discerned.

In view of the fact that tubal occlusion may give no evidence of its presence, insufflation and salpingography are essential procedures in the initial resolution of the cases into the two groups. There is much to be said for using these tests as complementary procedures, reserving X-ray examination to confirm a negative insufflation and to determine the site of any tubal occlusion.

Both procedures are essentially diagnostic, and it is better to regard them in this light; their use as primary therapeutic measures is likely to lead to many disappointments, and will tend to bring them into discredit.

In general, it may be said that if the tubes are demonstrated patent conception will ultimately occur in about half the cases. If careful steps can be taken to exclude the other causes of sterility, azoospermia, anovulatory cycles, endometrial tuberculosis, then the proportion of pregnancies following positive tests will very likely be still higher. Even if neither test has any therapeutic value, a view for which some support can be found, there is no doubt that the opportunity they afford of reassuring the patient that there is no fundamental bar to conception is itself of considerable therapeutic value.

The general problem of childlessness is indeed very similar to that presented by cases of recurrent abortion, which can be similarly divided into two main categories, one in which a cause can be discerned, and the prognosis is poor unless the cause is remediable, one in which no cause can be found, and the prognosis is good because the unknown causes are transient. In the case of childlessness the special place of insufflation and hysterosalpingography is that of key procedures in the initial division of the cases. They do not stand by themselves, but should be used along with the other methods of investigation.

My thanks are due to Dr. P. H. Whitaker for his help and advice on the radiological aspects of the problem.

\section{REFERENCES}

SHARMAN, A., Journ. Obstet and Gynaec. Brit. Emp., I944, LI, No. $2,85$.

WITWER, E. R., CUSHMAN, H. P., LEUCUTIA, T., Amer. Journ. Roentgen, I930, XXIII. I25.

GREEN-ARMYTAGE, V. B., Journ. Obst. and Gynaec. Brit. Emp., I943, L, 23.

WILLIAM S. ROHAN, Brit. Jour. Radiol., I944, XVII. I3.

DIBLE, J. H., HEWER, T. F., ROSS, A O. F., WALSH, C. H., Lancet, r938, Feb. 5 th, 3 I 3.

RUBIN, I. C., Amer. Journ. Obstet. and Gynaec., I939, XXXVII, 394. 